\title{
Mental Health and Psychosocial Problems of Medical Health Workers during the COVID-19 Epidemic in China
}

\author{
Wen-rui Zhang ${ }^{a}$ Kun Wang ${ }^{a, b}$ Lu Yinc ${ }^{c}$ Wen-feng Zhao ${ }^{a}$ Qing Xue ${ }^{a}$ Mao Peng ${ }^{a}$ \\ Bao-quan Min ${ }^{a}$ Qing Tiand Hai-xia Leng ${ }^{a}$ Jia-lin Du ${ }^{a}$ Hong Chang ${ }^{a}$ Yuan Yang ${ }^{e}$ Wei Li $^{f}$ \\ Fang-fang Shangguang ${ }^{9}$ Tian-yi Yan ${ }^{\text {h }}$ Hui-qing Dong ${ }^{a}$ Ying Han ${ }^{a}$ Yu-ping Wanga, ${ }^{a, i, j}$ \\ Fiammetta Cosci ${ }^{k}$ Hong-xing Wang ${ }^{a, i, j, l}$ \\ ${ }^{a}$ Division of Neuropsychiatry and Psychosomatics, Department of Neurology, Xuanwu Hospital, Capital Medical \\ University, Beijing, China; ${ }^{b}$ Department of Neurology, Beijing Puren Hospital, Beijing, China; ' ${ }^{\mathrm{C}}$ Medical Research and \\ Biometrics Center, National Center for Cardiovascular Diseases, Beijing, China; ${ }^{d}$ Beijing Institute of Brain Disorders, \\ Capital Medical University, Beijing, China; ${ }^{e}$ Department of Psychiatry, Tongji Hospital, Tongji Medical College, \\ Huazhong University of Science and Technology, Wuhan, China; ${ }^{f}$ Department of Neurology, The 3rd People's \\ Hospital of Chengdu, Chengdu, China; ${ }^{9}$ School of Psychology, Capital Normal University, Beijing, China; ${ }^{\mathrm{h}} \mathrm{School}$ of \\ Life Sciences, Beijing Institute of Technology, Beijing, China; 'Beijing Key Laboratory of Neuromodulation, Beijing, \\ China; ${ }^{j}$ Center of Epilepsy, Beijing Institute for Brain Disorders, Capital Medical University, Beijing, China; ${ }^{\mathrm{k}} \mathrm{Clinical}$ \\ Psychology, Department of Health Sciences, University of Florence, Florence, Italy; 'Beijing Psychosomatic Disease \\ Consultation Center, Xuanwu Hospital, Capital Medical University, Beijing, China
}

\section{Keywords}

Corona virus · COVID-19 · Health workers · Mental health · Psychosocial problems

\begin{abstract}
Objective: We explored whether medical health workers had more psychosocial problems than nonmedical health workers during the COVID-19 outbreak. Methods: An online survey was run from February 19 to March 6, 2020; a total of 2,182 Chinese subjects participated. Mental health variables were assessed via the Insomnia Severity Index (ISI), the Symptom Check List-revised (SCL-90-R), and the Patient Health Questionnaire-4 (PHQ-4), which included a 2-item anxiety scale and a 2-item depression scale (PHQ-2). Results: Compared with nonmedical health workers $(n=1,255)$, medical health workers $(n=927)$ had a higher prevalence of in-
\end{abstract}

somnia (38.4 vs. $30.5 \%, p<0.01$ ), anxiety (13.0 vs. $8.5 \%, p<$ 0.01 ), depression (12.2 vs. $9.5 \% ; p<0.04$ ), somatization (1.6 vs. $0.4 \% ; p<0.01)$, and obsessive-compulsive symptoms (5.3 vs. $2.2 \% ; p<0.01)$. They also had higher total scores of ISI, GAD-2, PHQ-2, and SCL-90-R obsessive-compulsive symptoms ( $p \leq 0.01$ ). Among medical health workers, having organic disease was an independent factor for insomnia, anxiety, depression, somatization, and obsessive-compulsive symptoms ( $p<0.05$ or 0.01 ). Living in rural areas, being female, and being at risk of contact with COVID-19 patients were the most common risk factors for insomnia, anxiety, obsessive-compulsive symptoms, and depression $(p<0.01$ or 0.05). Among nonmedical health workers, having organic disease was a risk factor for insomnia, depression, and obses-

Wen-rui Zhang, Kun Wang, and Lu Yin contributed equally to this work.

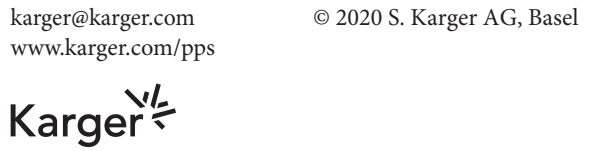


sive-compulsive symptoms ( $p<0.01$ or 0.05 ). Conclusions: During the COVID-19 outbreak, medical health workers had psychosocial problems and risk factors for developing them. They were in need of attention and recovery programs.

(c) 2020 S. Karger AG, Basel

\section{Introduction}

The coronavirus disease 2019 (COVID-19) outbreak is a pandemic [1] in which a coronavirus has been identified as the cause of an outbreak of respiratory illness. It was first detected in Wuhan, China [2], but COVID-19 is becoming an increasing public event being a rapid epidemic $[3,4]$. According to the official website of the World Health Organization [5], as of March 7, 2020, more than 100,000 people have been confirmed to have a COVID-19 infection globally. Many accomplishments on COVID-19, including virus information, clinical features, and diagnosis have been achieved, but no effective treatment is available yet $[2,6-8]$.

Medical health workers are first-line fighters treating patients with COVID-19. Every day, they face a high risk of being infected and are exposed to long and distressing work shifts to meet health requirements. In brief, they are exposed to a protracted source of distress which may exceed their individual coping skills, being, according to a clinimetric definition [9], in allostatic load, which is likely to result in overload with protracted time [9].

Despite messages mentioning that medical health workers' mental health should be emphasized during the campaign against COVID-19 [10-12], no research on mental health problems in medical health workers after the maximum point of the COVID-19 epidemic in China has been reported.

Since Chinese medical health workers have been exposed to a persistent source of distress, the aim of the present research was to outline its psychological manifestations. For this purpose, the prevalence and potential factors contributing to insomnia, anxiety, depression, obsessive-compulsive symptoms, somatization symptoms, and phobic anxiety were detected.

\section{Materials and Methods}

\section{Design, Participants, and Procedure}

This is a cross-sectional study performed via an online survey run from February 19 to March 6, 2020. The study was performed 8 weeks after the COVID-19 epidemic outbreak in Wuhan [2]. This survey period corresponded to the reducing stage after the maximum point of the COVID-19 epidemic outbreak in China [13], i.e., the highest vulnerability period after the great distress.

Persons in the nation with at least 16 years of age were welcome to join in the online survey via the Wenjuanxing platform (https:// www.wjx.cn/m/58475441.aspx).

The online survey included questions on sociodemographic and clinical variables. A simple math question (i.e., 21-7 =?) was added at the end for ensuring the quality and completeness of the questionnaire. Thus, participants who had not completed the survey received from the online platform a warning on unanswered questions when they did the math question. The online platform did not give warnings to those who gave up. As a result, participants were those who completed all questions of the online survey.

\section{Measurements}

Demographic data, i.e., sex, age, occupation (medical health workers, i.e., medical doctors and nurses, and nonmedical health workers, excluding nonmedical personnel working in hospitals/ medical institutions), marital status (i.e., married, unmarried, divorced, and widowed), living area (i.e., urban and rural), living with families (yes or no), education status ( $\leq 9$ years, i.e. junior high school and lower, $>9$ years, i.e. senior high school and higher) were collected via ad hoc questions as well as the information of a risk of contact with COVID-19 patients in hospitals. Participants were also asked whether they have had insomnia or psychiatric disorders prior to COVID-19 (those who replied positively were automatically excluded by the platform) and whether they were having organic diseases (the question was "Do you currently have any organic disease? [diagnosed by medical examination in the hospital]"). In addition, insomnia, anxiety, depression, somatization, obsessive-compulsive symptoms, and phobic anxiety were assessed.

Insomnia was assessed via the Insomnia Severity Index (ISI), a 7-item self-report index assessing the severity of initial, middle, and late insomnia [14]. An ISI total score $>8$ indicates that insomnia is present [15]. The item "Since the outbreak, how long (in minutes) did you usually take to fall asleep each night?" was added to assess the degree of sleep onset latency in medical health workers. This item was rated as $0,1,2$, and 3 (i.e., $\leq 15,16-30,31-60$, and $>60 \mathrm{~min}$, respectively).

Anxious and depressive symptoms were assessed via the $\mathrm{Pa}$ tient Health Questionnaire-4 (PHQ-4) [16], which is an ultra-brief self-report questionnaire with a 2-item anxiety scale, named Generalized Anxiety Disorder 2-item (GAD-2), and a 2-item depression scale, named Patient Health Questionnaire 2-item (PHQ-2). In screening of depression and anxiety, a cutoff $\geq 3$ in GAD- 2 and PHQ-2 is recommended [16].

Somatic symptoms, obsessive-compulsive symptoms, and phobic anxiety were measured via the Symptom Check List-90revised (SCL-90-R) $[17,18]$, a 90-item self-report scale with items rated on a 5-point Likert scale (from 0 "not at all" to 4 "extremely"). Subscale scores $\geq 2$ indicate potential psychological issues [19].

The Chinese versions of ISI [20], PHQ-2 [21], GAD-2 [22], and SCL-90-R [18] were used; they were validated and showed excellent psychometric properties.

Statistical Analyses

$\chi^{2}$ tests were used to compare group differences of categorical variables. Mann-Whitney tests were used to compare independent groups on continuous variables nonnormally distributed. 
Table 1. Sociodemographic characteristics in medical health workers versus nonmedical health workers

\begin{tabular}{|c|c|c|c|c|}
\hline Characteristics & $\begin{array}{l}\text { Total } \\
(n=2,182)\end{array}$ & $\begin{array}{l}\text { Medical } \\
\text { health workers } \\
(n=927)\end{array}$ & $\begin{array}{l}\text { Nonmedical } \\
\text { health workers } \\
(n=1,255)\end{array}$ & $\begin{array}{l}p \text { value } \\
\chi^{2} \text { test }\end{array}$ \\
\hline Sex, \% (n) & & & & $<0.01$ \\
\hline Male & $35.8(781)$ & $26.9(249)$ & $42.4(532)$ & \\
\hline Female & $64.2(1,401)$ & $73.1(678)$ & $57.6(723)$ & \\
\hline $18-60$ years & $96.3(2,101)$ & $98.4(912)$ & $94.7(1,189)$ & \\
\hline$>60$ years & $3.2(70)$ & $1.4(13)$ & $4.5(57)$ & \\
\hline Marital status, \% (n) & & & & 0.70 \\
\hline Single & $18.0(392)$ & $18.3(170)$ & $17.7(222)$ & \\
\hline Married & $82.0(1,790)$ & $81.7(757)$ & $82.3(1,033)$ & \\
\hline Living areas, $\%(n)$ & & & & $<0.01$ \\
\hline Yes & $88.0(1,920)$ & $83.6(775)$ & $91.2(1,145)$ & \\
\hline Education, \% (n) & & & & $<0.01$ \\
\hline$\leq 9$ years & $5.5(120)$ & $0.3(3)$ & $9.3(117)$ & \\
\hline$>9$ years & $94.5(2,062)$ & $99.7(924)$ & $90.7(1,138)$ & \\
\hline Risk of contact with COVID-19 patients in hospitals, \% ( $n$ ) & & & & - \\
\hline No & - & $85.1(789)$ & - & \\
\hline Yes & - & $14.9(138)$ & - & \\
\hline Having organic diseases, $\%(n)$ & & & & 0.78 \\
\hline No & $89.0(1,942)$ & $88.8(823)$ & $89.2(1,119)$ & \\
\hline Yes & $11.0(240)$ & $11.2(104)$ & $10.8(136)$ & \\
\hline Insomnia Severity Index (ISI), \% (n) & & & & $<0.01$ \\
\hline No clinically significant insomnia (total ISI score: $0-7$ ) & $66.1(1,443)$ & $61.6(571)$ & $69.5(872)$ & \\
\hline No & $89.6(1,954)$ & $87.0(806)$ & $91.5(1,148)$ & \\
\hline Yes & $10.4(228)$ & $13.0(121)$ & $8.5(107)$ & \\
\hline Depression, \% (n) & & & & 0.04 \\
\hline No & $89.4(1,950)$ & $87.8(814)$ & $90.5(1,136)$ & \\
\hline Yes & $10.6(232)$ & $12.2(113)$ & $9.5(119)$ & \\
\hline Somatization symptoms, \% (n) & & & & $<0.01$ \\
\hline No & $99.1(2,162)$ & $98.4(912)$ & $99.6(1,250)$ & \\
\hline Yes & $0.9(20)$ & $1.6(15)$ & $0.4(5)$ & \\
\hline Obsessive-compulsive symptoms, \% (n) & & & & $<0.01$ \\
\hline No & $96.5(2,105)$ & $94.7(878)$ & $97.8(1,227)$ & \\
\hline Yes & $3.5(77)$ & $5.3(49)$ & $2.2(28)$ & \\
\hline Phobic anxiety, \% (n) & & & & 0.11 \\
\hline No & $97.1(2,119)$ & $96.4(894)$ & $97.6(1,225)$ & \\
\hline Yes & $2.9(63)$ & $3.6(33)$ & $2.4(30)$ & \\
\hline
\end{tabular}

COVID-19, the coronavirus disease 2019. 
Multivariate logistic regression analyses were performed using stepwise variable selection, and all variables were entered into the model to explore independent influence for different risk dimensions, such as insomnia, anxiety, depression, somatization, obsessive-compulsive symptoms, and phobic anxiety. Subgroup analyses were performed for medical and nonmedical health workers.

All hypotheses were tested at a significance level of 0.05 .

Data analyses were run via SAS statistical software, version 9.4 (SAS Institute Inc.).

\section{Results}

Nationwide, a total of 2,182 participants from China (see online supplement 1; for all online suppl. material, see www.karger.com/doi/10.1159/000507639) completed the survey.

Table 1 presents sociodemographic features of the whole sample and compared 927 medical health workers (680 medical doctors and 247 nurses) to 1,255 nonmedical health workers. Medical health workers showed higher prevalence rates of insomnia ( 38.4 vs. $30.5 \%, p<0.01$ ), anxiety ( 13.0 vs. $8.5 \%, p<0.01)$, depression ( 12.2 vs. $9.5 \%$; $p=0.04$ ), somatization (1.6 vs. $0.4 \% ; p<0.01$ ), and obsessive-compulsive symptoms ( 5.3 vs. $2.2 \% ; p<0.01)$ than nonmedical health workers. Medical health workers also had higher total scores of ISI $(p<0.01)$, GAD-2 $(p<0.01)$, PHQ-2 $(p=0.01)$, and on the SCL-90-R obsessive-compulsive symptom scale $(p<0.01)$ than nonmedical health workers. Each item of ISI $(p<0.01$ or $p<0.05)$, GAD-2 $(p<0.01)$, and PHQ-2 $(p=$ 0.01 ) was significantly elevated in medical health workers compared with nonmedical health workers. On the SCL90-R obsessive-compulsive symptom scale, 6 of the 10 items had higher scores in medical health workers than in nonmedical health workers. In the SCL-90-R somatization symptoms scale, 3 of 12 items, including questions 1 (headaches) $(p=0.01), 4$ (faintness or dizziness) $(p<0.01)$, and 48 (trouble getting your breath) $(p<0.01)$, had higher scores in medical health workers than in nonmedical health workers. No difference on phobic anxiety between both groups was found (Table 2).

The multivariate logistic regression analyses (Table 3 ) showed that living in rural areas (odds ratio [OR], 2.18, $95 \%$ confidence interval $[\mathrm{CI}], 1.42-3.35 ; p<0.01)$, being at risk of contact with COVID-19 patients in hospitals (OR, 2.53; 95\% CI, 1.74-3.68; $p<0.01$ ), and having organic diseases (OR, 3.39; 95\% CI, 2.20-5.22; $p<0.01$ ) were risk factors for insomnia among medical health workers, while only having organic diseases (OR, 2.23; $95 \%$ CI, $1.55-3.20 ; p<0.01$ ) was a risk factor for the nonmedical health workers.

COVID-19 Epidemic: Psychosocial Problems in Medical Health Workers
Four variables were independently associated with anxiety risk among medical health workers: being female (OR, 1.80, 95\% CI, 1.10-2.95; $p=0.02$ ), living in rural areas (OR, 1.88; 95\% CI, 1.09-3.21; $p=0.02$ ), being at risk of contact with COVID-19 patients in hospitals (OR, 2.06; 95\% CI, $1.28-3.32 ; p<0.01)$, and having organic diseases (OR, 2.85; 95\% CI, 1.73-4.68; $p<0.01$ ).

In depression models, being female (OR, 1.85, 95\% CI, $1.11-3.08 ; p=0.02)$ and having organic diseases (OR, 2.51 ; 95\% CI, 1.51-4.18; $p<0.01)$ were selected as independent factors among medical health workers, while having organic diseases (OR, 1.90; 95\% CI, 1.13-3.20; $p<$ 0.01 ) and living with families (OR, 0.36; 95\% CI, $0.21-$ $0.59 ; p<0.01)$ were predictors for depression among nonmedical health workers.

For obsessive-compulsive symptoms, medical health workers had 3 risk factors: living in rural areas (OR, 2.49; $95 \%$ CI, 1.21-5.11; $p=0.01$ ), being at risk of contact with COVID-19 patients in hospitals (OR, 3.27; 95\% CI, 1.75$6.11 ; p<0.01$ ), and having organic diseases (OR, 2.24; $95 \%$ CI, $1.07-4.71 ; p=0.03)$. On the other hand, nonmedical health workers had organic diseases (OR, 2.84; $95 \% \mathrm{CI}, 1.18-6.80 ; p=0.02$ ) as only risk factor.

Regarding somatization symptoms, medical health workers had 2 risk factors: living in rural areas (OR, 4.78; $95 \% \mathrm{CI}, 1.55-14.76 ; p=0.01)$ and having organic diseases (OR, 7.89; 95\% CI, 2.75-22.62; $p<0.01$ ). No risk factors were found for nonmedical health workers.

\section{Discussion}

Medical health workers during the COVID-19 epidemic had high prevalence rates of severe insomnia, anxiety, depression, somatization, and obsessive-compulsive symptoms. They also had risk factors for developing insomnia, anxiety, depression, obsessive-compulsive symptoms, and somatization. Thus, the presence of these symptoms in addition to the life status of daily fighting against COVID-19 suggests that they must cope with psychological distress and are at risk of allostatic overload [9]. Indeed, according to clinimetric criteria, allostatic overload can be diagnosed in the presence of a current identifiable source of distress in the form of recent life events and/or chronic stress; the stressor is judged to tax or exceed the individual coping skills when its full nature and full circumstances are evaluated. In addition, the stressor is associated with difficulty in falling asleep, restless sleep, early morning awakening, lack of energy, dizziness, generalized anxiety, irritability, sadness, demoralization; significant impairment in 
Table 2. Psychological manifestations of medical health workers versus nonmedical health workers

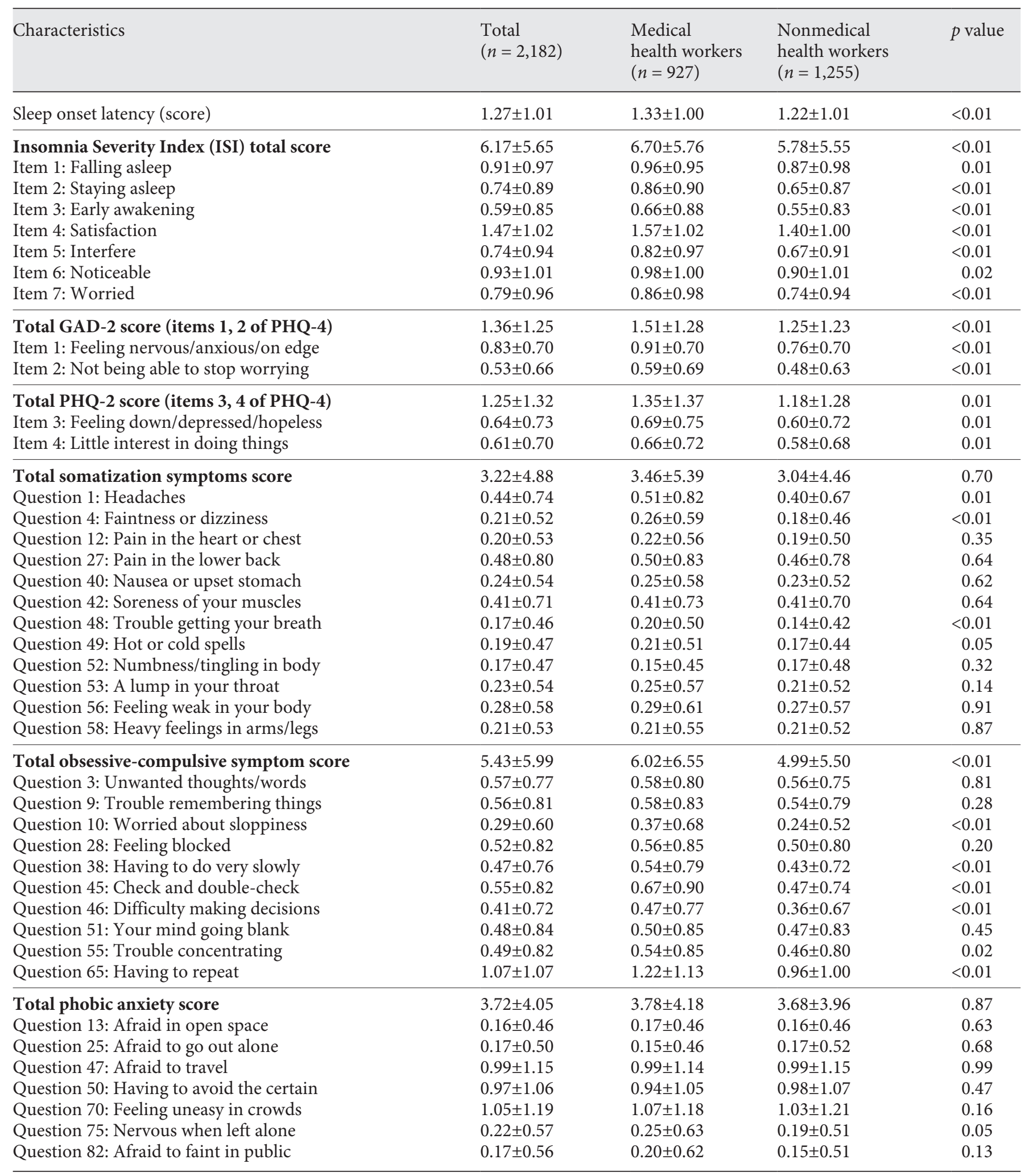

GAD-2, Generalized Anxiety Disorder 2-item; PHQ-4, Patient Health Questionnaire-4; PHQ-2, Patient Health Questionnaire 2 items. Mann-Whitney test for independent samples. 
Table 3. Outcomes of psychological manifestations

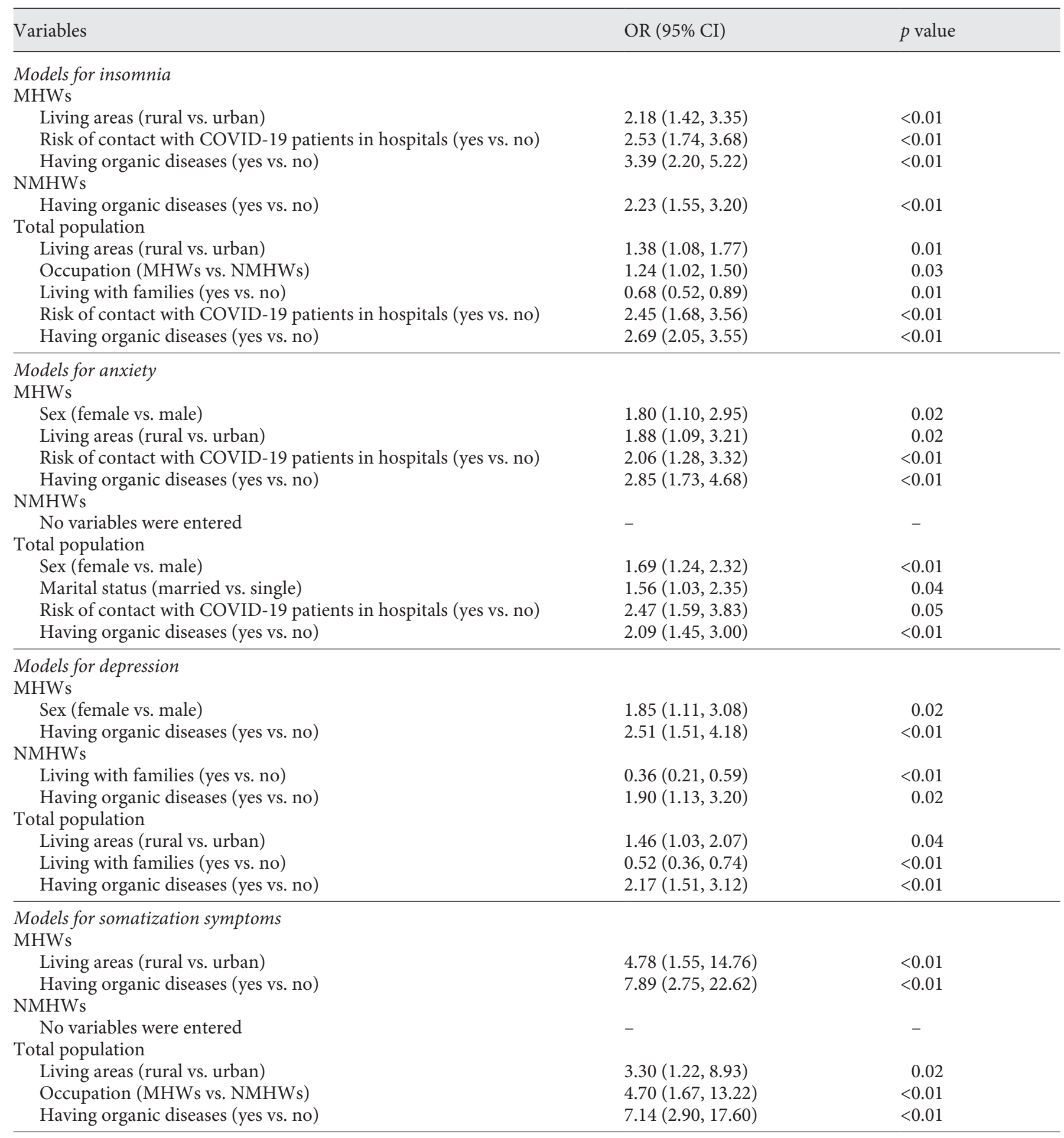


Table 3 (continued)

\begin{tabular}{llr}
\hline Variables & OR (95\% CI) & $p$ value \\
\hline Models for obsessive-compulsive symptoms & & \\
MHWs & $2.49(1.21,5.11)$ & 0.01 \\
$\quad$ Living areas (rural vs. urban) & $3.27(1.75,6.11)$ & 0.01 \\
$\quad$ Risk of contact with COVID-19 patients in hospitals (yes vs. no) & $2.24(1.07,4.71)$ & 0.03 \\
$\quad$ Having organic diseases (yes vs. no) & $2.84(1.18,6.80)$ & 0.02 \\
NMHWs & $1.85(1.10,3.10)$ & $<0.01$ \\
$\quad$ Having organic diseases (yes vs. no) & $3.38(1.81,6.29)$ \\
Total population & $2.43(1.38,4.28)$ \\
$\quad$ Occupation (MHWs vs. NMHWs) & \\
$\quad$ Risk of contact with COVID-19 patients in hospitals (yes vs. no) & $<0.01$ \\
$\quad$ Having organic diseases (yes vs. no) & \\
\hline Models for phobic anxiety & - \\
MHWs & \\
$\quad$ No variables were entered & - \\
$\quad$ NMWs & \\
$\quad$ To variables were entered & \\
$\quad$ Sex (female vs. male) & $2.19(1.18,4.05)$ \\
\hline
\end{tabular}

OR, odds ratio; CI, confidence interval; MHWs, medical health workers; COVID-19, the coronavirus disease 2019; NMHWs, nonMHWs. Multivariate logistic regression analyses using stepwise variable selection.

social or occupational functioning; and feeling overwhelmed by the demands of everyday life [9].

The reasons for the psychological distress to which medical health workers were exposed might be related to the many difficulties of being safe at work, such as the initially insufficient understanding of the virus, the lack of prevention and control knowledge, the long-term workload, the high risk of exposure to patients with COVID-19, the shortage of medical protective equipment $[13,23]$, the lack of getting rest [24], and the exposure to critical life events [25], such as death. Exemplifications of such a distress are: (1) 16 of the 100 nurses at the Pohang Medical Center in North Gyeongsang Province resigned due to overwork among the COVID-19 epidemic [26]; (2) >3,000 medical health workers in (Wuhan) Hubei Province were infected with COVID-19 at a very early stage (before and in January of 2020). Later, with continuously updated guidelines on how to handle the patients with COVID-19 [27], with rest in shifts for medical staff, with rapid supply of medical protective items (including masks, glasses, and suits), and with training on the Novel Coronavirus Infection Pneumonia Diagnosis and Treatment Plan for all medical staff [24], no doctors have been infected with COVID-19 among about 40,000 medical personnel from the nation supporting Hubei medical services [24]; and (3) as of the 20th March, 5 medical health workers in one hospital of Wuhan died due to being infected with COVID-19 [28].

Our report found potential risk factors for medical health workers to develop insomnia, anxiety, depression, obsessive-compulsive symptoms, and somatization. Undoubtedly, these risk factors might endure allostatic overload and favor the development of psychopathology, including chronic insomnia [29]. Independent factors (i.e., currently having organic disease, living in rural areas, being at risk of contact with COVID-19 patients in hospitals, or being female) were common risk factors for insomnia, anxiety, depression, and obsessive-compulsive symptoms among medical health workers. When faced with the same COVID-19 during the fight against the epidemic, medical health workers in rural areas might worry about being infected due to a different working place involving different medical skills and medical conditions. In contrast, the medical conditions in urban areas were often much better. Thus, different directions on caring for the medical health workers might be possible.

Adequate working conditions and recovery programs, i.e., programs favoring activities required to ensure the best physical, mental, and social conditions so that medical workers may progress towards an optimal state of health [30], seem necessary. This may support medical staff in adapting to the working environment quickly and 
maintain a better mental and health balance to be able to work. Lowering job demands and workload [31], while increasing job control and reward might help to protect medical health workers. Individual interventions adequate for medical staff in the current situation, where they wear medical protective equipment which cannot be removed during work time, are still unknown. Story sharing [32] would be important as well as reinforcing the positive assets of persons [33]. Simple, easy, practical methods are needed. Electronic devices, such as mobile phones and computers, may help.

The present study has limitations. First, a cross-sectional design was applied although a longitudinal approach might help verifying whether allostatic overload develops (exhaustion may ensue after some time) and whether psychiatric disorders, especially posttraumatic stress disorder, might occur with the COVID-19 progression. Second, psychological assessment was based on an online survey and on self-report tools. The use of clinical interviews is encouraged in future studies to draw a more comprehensive assessment of the problem. Third, it is not possible to assess the participation rate since it is unclear how many subjects received the link for the survey.

In conclusion, a higher prevalence of psychological symptoms was found among medical health workers during COVID-19 as well as risk factors for them. Medical health workers are in need of health protection and adequate working conditions, e.g., provision of necessary and sufficient medical protective equipment, arrangement of adequate rest, as well as recovery programs aimed at empowering resilience and psychological well-being [34].

\section{Acknowledgment}

The authors would like to thank all participants for their time and excellent cooperation.

\section{Statement of Ethics}

All participants provided their online informed consent. The study was approved by the local ethics committee on human research.

\section{Disclosure Statement}

The authors declare that they have no conflicts of interests.

\section{Funding Sources}

H.W. was supported by grants from the National Natural Science Foundation of China (81771862), National Key R\&D Program of China (2017YFC1310001, 2016YFC1307000), Beijing Municipal Science and Technology Project (Z171100000117016), Beijing Natural Science Foundation (KZ201710025017), Beijing Municipal Hospital Research and Development Plan (PX2017069), and Beijing Hundred, Thousand, and Ten Thousand Talents Project (2017-CXYF-09). Y.W. was supported by the Beijing Key Clinical Speciality Excellence Project. The funding organizations had no role in the design and conduct of the study; collection, management, analysis, and interpretation of the data; preparation, review, or approval of the manuscript; and decision to submit the manuscript for publication.

\section{Author Contributions}

H.W.: conception and design; W.Z., K.W., L.Y., W.Z., Q.X., Y.Y., M.P., B.M., Q.T., H.L., J.D., H.C., W.L., F.S., T.Y., H.D., Y.H., Y.W., and H.W.: conduction; H.W. and L.Y.: statistical analysis; W.Z., K.W., W.Z., Q.X., and W.L.: administrative, technical, or material support; H.W.: drafting of the manuscript; and F.C. and H.W.: critical revision of the manuscript for important intellectual content. All authors read and approved the final paper.

\section{References}

1 World Health Organization [Internet]. Geneva: WHO characterizes COVID-19 as a pandemic [cited 2020 March 11]. Available from: https://www.who.int/emergencies/ diseases/novel-coronavirus-2019/events-asthey-happen.

2 Li Q, Guan X, Wu P, Wang X, Zhou L, Tong $\mathrm{Y}$, et al. Early transmission dynamics in $\mathrm{Wu}-$ han, China, of novel coronavirus-infected pneumonia. N Engl J Med. 2020 Mar;382(13): 1199-207.

3 Lancet. COVID-19: too little, too late? Lancet. 2020 March;395(10226):755

COVID-19 Epidemic: Psychosocial Problems in Medical Health Workers
4 Day M. Covid-19: surge in cases in Italy and South Korea makes pandemic look more likely. BMJ. 2020 Feb;368:m751.

5 World Health Organization [Internet]. Geneva: WHO statement on cases of COVID-19 surpassing 100000 [cited 2020 March 7]. Available from: https://www.who.int/newsroom/detail/07-03-2020-who-statement-oncases-of-covid-19-surpassing-100-00.

6 Guan WJ, Ni ZY, Hu Y, Liang WH, Ou CQ, He JX, et al.; China Medical Treatment Expert Group for Covid-19. Clinical characteristics of coronavirus disease 2019 in China. N Engl J Med.2020. DOI: 10.1056/NEJMoa20020322.
7 Zhou P, Yang XL, Wang XG, Hu B, Zhang L, Zhang W, et al. A pneumonia outbreak associated with a new coronavirus of probable bat origin. Nature. 2020 Mar;579(7798): 270-3.

8 Wang M, Cao R, Zhang L, Yang X, Liu J, Xu $\mathrm{M}$, et al. Remdesivir and chloroquine effectively inhibit the recently emerged novel coronavirus (2019-nCoV) in vitro. Cell Res. 2020 Mar;30(3):269-71.

9 Fava GA, McEwen BS, Guidi J, Gostoli S, Offidani E, Sonino N. Clinical characterization of allostatic overload. Psychoneuroendocrinology. 2019 Oct;108:94-101. 
10 Bao Y, Sun Y, Meng S, Shi J, Lu L. 2019-nCoV epidemic: address mental health care to empower society. Lancet. 2020 Feb; 395 (10224):e37-8.

11 Kang L, Li Y, Hu S, Chen M, Yang C, Yang BX, et al. The mental health of medical workers in Wuhan, China dealing with the 2019 novel coronavirus. Lancet Psychiatry. 2020 Mar; 7(3):e14.

12 Liu S, Yang L, Zhang C, Xiang YT, Liu Z, Hu $S$, et al. Online mental health services in China during the COVID-19 outbreak. Lancet Psychiatry. 2020 Apr;7(4):e17-8.

13 Guangming Online [Internet]. Beijing: Central Steering Group: Over 3,000 medical staff in Hubei were infected in the early stage of the epidemic, currently no infection reports among medical aid staff [cited 2020 March 6]. Available from: https://politics.gmw.cn/202003/06/content_33626862.htm. Chinese.

14 Morin CM, Belleville G, Bélanger L, Ivers H. The Insomnia Severity Index: psychometric indicators to detect insomnia cases and evaluate treatment response. Sleep (Basel). 2011 May;34(5):601-8.

15 Wong SY, Zhang DX, Li CC, Yip BH, Chan DC, Ling YM, et al. Comparing the effects of mindfulness-based cognitive therapy and sleep psycho-education with exercise on chronic insomnia: a randomised controlled trial. Psychother Psychosom. 2017;86(4): 241-53.

16 Löwe B, Wahl I, Rose M, Spitzer C, Glaesmer $\mathrm{H}$, Wingenfeld $\mathrm{K}$, et al. A 4-item measure of depression and anxiety: validation and standardization of the Patient Health Questionnaire-4 (PHQ-4) in the general population. J Affect Disord. 2010 Apr;122(1-2):86-95.

17 Wang ZY. Symptom Check List (SCL-90). Shanghai Jingshen Yixue. 1984;2(2):68-70. Chinese.

18 Chen S, Li L. Re-testing reliability, validity, and norm applicability of SCL-90. Chin J Nerv Ment Dis. 2003;29(5):323-7. Chinese.
19 Chen X, Li P, Wang F, Ji G, Miao L, You S. Psychological results of 438 patients with persisting gastroesophageal reflux disease symptoms by Symptom Checklist 90-Revised Questionnaire. Euroasian J Hepatogastroenterol. 2017 Jul-Dec;7(2):117-21.

20 Zhang B, Zhang L, Chen S, Luo X, Dhirendra $\mathrm{P}$, Lin $\mathrm{Q}$, et al. The effect of e-aid cognitive behavioral therapy in treating chronic insomnia disorder: an open-label randomized controlled trial. Zhonghua Jing Shen Ke Za Zhi. 2019 Dec;52(6):373-8. Chinese.

21 Wang L, Lu K, Li J, Sheng L, Ding R, Hu D. Value of patient health questionnaires (PHQ)-9 and PHQ-2 for screening depression disorders in cardiovascular outpatients. Zhonghua Xin Xue Guan Bing Za Zhi. 2015 May 2015;43(5):428-431. Chinese.

22 Wang L, Lu K, Wang C, Sheng L, Hu D, Ding R. Reliability and validity of GAD-2 and GAD-7 for anxiety screening in cardiovascular disease clinic. Sichuan Mental Health. 2014;27(3):198-201. Chinese.

23 World Health Organization [Internet]. Geneva: Shortage of personal protective equipment endangering health workers worldwide [cited 2020 March 3]. Available from: https:// www.who.int/news-room/detail/0303-2020-shortage-of-personal-protectiveequipment-endangering-health-workersworldwide.

24 SINAnews [Internet]. Beijing: In January, Hubei had more than 3,000 medical infections, and the Wuhan Health and Medical Committee reported "None" for half a month [cited 2020 March 6]. Available from: https:// news.sina.com.cn/o/2020-03-06/dociimxyqvz8395569.shtml. Chinese.

25 Theorell T. Evaluating life events and chronic stressors in relation to health: stressors and health in clinical work. Adv Psychosom Med. 2012;32:58-71.
26 The Telegraph [Internet]. London: Coronavirus: Doctors collapse from exhaustion as virus spreads through South Korea [cited 2020 March 3]. Available from: https://www.telegraph.co.uk/news/2020/03/03/coronavirusdoctors-collapse-exhaustion-virus-spreadssouth/.

27 Chinanews [Internet]. Beijing: Two departments issue the novel coronavirus infection pneumonia diagnosis and treatment plan. (trial version 7) [cited 2020 March 4]. Available from: http://www.chinanews.com/gn/ 2020/03-04/9113100.shtml. Chinese.

28 China.com. [Internet]. Beijing: Liu Li, Wuhan Central Hospital, infected with COVID-19, died, totally five died in the hospital. 2020 [cited 2020 March 20]. Available from: https://news.china.com/socialgd/10000169/ 20200320/37949631.html. Chinese.

29 Wang HX, Wang L, Zhang WR, Xue Q, Peng $\mathrm{M}$, Sun ZC, et al. Effect of transcranial alternating current stimulation for the treatment of chronic insomnia: a randomized, doubleblind, parallel-group, placebo-controlled clinical trial. Psychother Psychosom. 2020; 89(1):38-47.

30 Sonino N, Fava GA. Rehabilitation in endocrine patients: a novel psychosomatic approach. Psychother Psychosom. 2007;76(6): 319-24.

31 Aronsson G, Theorell T, Grape T, Hammarström A, Hogstedt C, Marteinsdottir I, et al. A systematic review including meta-analysis of work environment and burnout symptoms. BMC Public Health. 2017 Mar;17(1):264.

32 Cummings J. Sharing a traumatic event: the experience of the listener and the storyteller within the dyad. Nurs Res. 2011 Nov-Dec; 60(6):386-92.

33 Fava GA. Well-Being Therapy: Treatment Manual and Clinical appliatios. Basel: Kager; 2016. https://doi.org/10.1159/isbn.978-3-31805822-2.

34 Fava GA, Cosci F, Sonino N. Current psychosomatic practice. Psychother Psychosom. 2017;86(1):13-30. 